\title{
Expression of selected angiogenesis-related small microRNAs in patients with abnormally increased secretion of glucocorticoids
}

\author{
Miłosz P. Kawa' ${ }^{1}$ Anna Sobuś1, Zofia Litwińska', Lilianna Osowicz-Korolonek ${ }^{2}$, Aneta Cymbaluk-Płoska ${ }^{3}$, \\ Iwona Stecewicz ${ }^{4}$, Edyta Zagrodnik', Hanna Romanowska ${ }^{4}$, Mieczysław Walczak , Anhelli Syrenicz², \\ Bogusław Machaliński \\ ${ }^{1}$ Department of General Pathology, Pomeranian Medical University, Szczecin, Poland \\ ${ }^{2}$ Department of Endocrinology, Metabolic Diseases, and Internal Diseases, Pomeranian Medical University, Szczecin, Poland \\ ${ }^{3}$ Department of Gynaecological Surgery and Gynaecological Oncology, Pomeranian Medical University, Szczecin, Poland \\ ${ }^{4}$ Department of Paediatrics, Endocrinology, Diabetology, Metabolic Diseases, and Cardiology of Developmental Age, Pomeranian \\ Medical University, Szczecin, Poland
}

\begin{abstract}
Introduction: Higher cortisol levels are associated with cardiovascular morbidity and mortality in the elderly, partially resulting from biologic effects of glucocorticoids (GCs) on endothelial cells observed in an experimental setting. These features are replicated in patients with endogenous GC excess (Cushing's syndrome) or with exogenous hypercortisolism due to excessive pharmacological application of GCs. Both groups present also an increased cardiovascular disease event rate. GCs may also adversely influence recovery after myocardial infarction. Recently it was proposed that microRNAs (miRNAs) - small noncoding RNAs functioning as antisense regulators of gene expression by targeting mRNA - may have a central role in regulating endothelial function through multiple mechanisms. Thus, the purpose of this study was to evaluate the effects of chronic GC excess on the expression of selected endothelium-controlling miRNAs expressed in nucleated cells circulating in peripheral blood (PBNCs) of patients with endogenous hypercortisolism either due to corticotrophin independent or corticotrophin dependent Cushing's syndrome (CS).

Material and methods: Peripheral blood nuclear cells were collected from 35 healthy subjects and 31 patients with endogenous hypercortisolism as a source of miRNAs. A self-validated individual quantitative RT-PCR study was then performed to evaluate the expression levels of selected miRNAs in PBNCs. Additionally, endothelin-1 (ET-1) expression in peripheral blood was assessed with respect to endothelial dysfunction using Western blotting.

Results: The ET-1 expression levels in CS were higher than in controls, confirming endothelial dysfunction in the CS group. Furthermore, miRNA analysis revealed a significantly decreased intracellular expression of selected endothelium-related miRNAs in patients with endogenous hypercortisolism, including miRNA-17-5p, miRNA-126-3p, and miRNA-126-5p, compared to controls. In contrast, two other angiogenic miRNAs, miRNA-150-5p and miRNA-223-3p, were significantly upregulated compared to controls.

Conclusions: Cardiovascular events related to hypercortisolism remain a challenging problem in medical practice. This study has demonstrated that the chronic excess of GCs in endogenous CS might induce significant dysregulation of selected miRNAs involved in the control of endothelium biology. However, the lack of knowledge about specific miRNA expression postpones the full understanding of the biological roles of such miRNAs in hypercortisolism. Moreover, dysregulated miRNAs seem to be promising targets for further research, especially to search for potential therapies for several GC-induced cardiovascular complications. (Endokrynol Pol 2019; 70 (6): 489-495)

Key words: endocrine diseases; Cushing's syndrome; cardiovascular abnormalities; microRNA
\end{abstract}

\section{Introduction}

Glucocorticoids (GCs) are used extensively to cure many diseases due to their anti-proliferative, immunosuppressive, and anti-inflammatory effects [1]. Thus, synthetic GCs are widely used with multiple indications and many administration forms used for both systemic and local disorders, including inflammatory and autoimmune diseases or haematological neoplasms. About $1 \%$ of the general population are using long-term GC therapy at any given time, due to the increased preva- lence of diseases requiring such therapy [2-3]. There is growing concern that markedly increased use of GCs can lead to supraphysiological GC exposure and may induce specific adverse cardio-metabolic complications, such as dyslipidaemia, adiposity and central obesity, decreased insulin sensitivity, hyperglycaemia and diabetes, inflammation, immune dysregulation and platelet activation, and finally hypertension and atherosclerosis followed by cardio-vascular derangements, such as increased risk of coronary artery disease, heart insufficiency, or stroke [4-5]. These exogenous 
GC-related complications have been strictly linked to increased cardio-vascular morbidity or mortality [4-5]. Nevertheless, there is no medical consensus on the best strategies for the prevention and detection of GC-induced complications, and available data on these adverse events are rather poor, with many of the clinical studies being inconclusive, resulting in the lack of optimal treatment strategies for patients affected with glucocorticoid-induced adverse cardio-metabolic disorders.

Nonetheless, some of these questions can be answered by analysing available data from patients with hypercortisolism in the course of endogenous Cushing's syndrome (CS), which is a model of the pathological state with extremely elevated cortisol levels. This is a condition characterised by excessive and continuous elevation of cortisol, usually caused by a pituitary or adrenal adenoma. It is known that ACTH and cortisol play a complex role in the regulation of carbohydrate, lipid, and protein metabolism, energy homeostasis, and the regulation of body fluids and body composition, all of them strongly related to vascular functions [6]. Thus, the excess of endogenous cortisol can lead to a variety of clinical manifestations, including cardio-metabolic disorders. Although long-term elevated cortisol is an important cardiovascular risk factor and higher cortisol levels were associated with a higher incidence of cardiovascular diseases (CVD), the molecular mechanisms responsible for GC-induced cardiovascular complications are not well established. Several studies have suggested the crucial role of nitric oxide (NO), eicosanoids, and oxidative stress together with renin-angiotensin system alterations in haemodynamic changes in the cardiovascular system of patients with hypercortisolism [7]. Glucocorticoid overexposure leads to alterations in the cardiovascular system structure and function also via vasoconstrictor factor endothelin-1 (ET-1). It was demonstrated that GCs induce ET-1 release, and suppress the activity of the NO system and thus cause vasoconstriction resulting in endothelial dysfunction [8]. Related studies have also shown that endothelial dysfunction is involved in other GC-induced vascular pathologies including hypertension [9], myocardial failure [10], dyslipidaemia [11], and atherosclerosis [12]. The coexisting persistent inflammatory environment further contributes to elevation of ET-1 [13], endothelial dysfunction, and impaired endothelium-dependent relaxation, all contributing to the pathogenesis of cardiovascular complications [14].

Importantly, the mechanisms for the development and progression of cardio-metabolic complications and the markers for remission and recurrence have not been sufficiently investigated yet. Several studies suggested that epigenetic regulation based on microRNA (miRNA) would have some importance in the development and progression of GC-related cardiovascular complications. These small non-coding RNAs control gene expression by targeting messenger RNA (mRNA) and disturb translation, and thus represent a major class of molecular regulators. An increasing number of specific miRNA expression signatures have been identified in CVD, and they have gained importance in recent years by helping diagnosis as biomarkers. It has also been demonstrated that miRNAs influence various genes known to be associated with angiogenesis. "AngioMIRs" are a set of miRNAs that play important roles in the regulation of angiogenic responses and endothelial cell (EC) functions. For instance, the miRNA-126 family, composed of two strands: miRNA-126-3p and miRNA-126-5p, is enriched in endothelial cells and is recognised as a leading regulator of angiogenesis and vascular integrity. The miRNA-126-5p strand promotes EC proliferation, improving re-endothelialisation after vascular injury [15]. In contrast, miRNA-126-3p interferes with the process of EC activation by inhibiting the expression of VCAM-1 molecules and impeding leukocyte adhesion to ECs and their transmigration [16]. The miRNA-126 cluster is a master regulator in endothelial dysfunction as well. The level of both miRNA-126 (miRNA-126-3p and miRNA-126-5p) were lowered in diabetic patients, and their expression was reduced in EC exposed to high glucose in vitro [17]. Low levels of miRNA-126 in diabetic EC are associated with decreased proliferation, migration, and NO production [18]. These observations suggest a strong role of the miRNA-126 cluster in the modulation of atherosclerosis development and vascular complications in diabetes. Other miRNAs shown to regulate angiogenesis, such as miRNA-17 cluster, are regulated by vascular shear stress and promote the growth of blood vessels [19]. Of note, blood levels of miRNA-17 and miRNA-126 were decreased in stable coronary artery disease. Regulation of EC function has also been demonstrated in the case of miRNA-150, which, secreted from activated monocytic cells, was able to enhance EC migration [20]. Other studies showed that levels of miRNA-150 were higher in patients with myocardial infarct and increased in response to inflammatory stimuli [21]. Likewise, miRNA-223, the expression of which is dependent on intracellular cholesterol levels, has also been reported to play a role in endothelium, especially due to the fact that miRNA-223 negatively regulates the inflammatory response by blocking the NLRP3 inflammasome and IL-1 $\beta$ production [22]. Altogether, it seems that the involvement of miRNAs in angiogenesis and endothelial cell function in cardiovascular system is substantial, making miRNAs presumably major governing forces in the pathogenesis of endothelial dysfunction developing 
in different CVD and cardio-metabolic complications found in other diseases.

The purpose of the study was to evaluate intracellular PBNC expression levels of five selected candidate "angioMIRs" that regulate various angiogenesis signalling pathways and endothelial dysfunction potentially involved in the pathogenesis of GC-induced cardiovascular complications in patients with Cushing's syndrome. We advocate that CS, usually affecting young and presumably healthy patients, is a good in vivo model to examine the effects of GC excess on the expression of angiogenesis-related microRNAs known to affect endothelial cell homeostasis in human vasculature, minimising other confounding factors including the disease itself, the dose and type of GC, as well as the compliance of the patients.

\section{Material and methods}

\section{Characteristics of the study group}

Thirty-one patients with hypercortisolism due to newly diagnosed endogenous Cushing's syndrome (CS), as a result of either adrenocortical or pituitary tumours [23], were recruited in the Department of Endocrinology of the Pomeranian Medical University in Szczecin, Poland. The control group consisted of 35 age-matched participants with no symptoms or signs of CS (defined as the absence of typical clinical features of CS). All of the enrolled subjects underwent a complete medical examination for statistical analyses. Data regarding medical history, current drug use, and smoking status were collected based on laboratory data, pathology tests, and other information, with a particular focus on heart and vascular conditions and existing arterial hypertension. The study adhered to the tenets of the Declaration of Helsinki, and approval was obtained from the Local Research Ethics Committee. Moreover, each patient provided written, informed consent for involvement in the study.

\section{Blood sample collection}

Venous blood samples $(\sim 7.5 \mathrm{~mL})$ collected in EDTA tubes were centrifuged $\left(2000 \mathrm{rpm}, 4^{\circ} \mathrm{C}, 10 \mathrm{~min}\right)$, and the plasma was stored at $-20^{\circ} \mathrm{C}$ to $-80^{\circ} \mathrm{C}$ until assayed. Next, the red blood cells were lysed using BD Pharm Lyse lysing buffer (BD Biosciences, San Jose, CA, USA) for $15 \mathrm{~min}$ at room temperature to isolate peripheral blood nuclear cells (PBNCs).

\section{Western blot analysis}

The total protein concentrations were determined using the Bradford protein assay (Sigma-Aldrich). Subsequently, the extracted proteins $(20 \mu \mathrm{g} /$ well) were separated on $14 \%$ gel (SDS-PAGE; Mini Protean Tetra Cell System; Bio-Rad). Fractionated proteins were transferred onto a 0.2- $\mu \mathrm{m}$ PVDF membrane (Bio-Rad), and the membranes were blocked with $3 \%$ bovine serum albumin (BSA) in buffer for one hour at RT. Endothelin-1 protein was detected using rabbit polyclonal IgG antibody (Santa Cruz Biotechnology) and goat anti-rabbit IgG with horseradish peroxidase as a secondary antibody (Santa Cruz Biotechnology). The membranes were developed with Western blot analysis (ECL Advance Western Blotting Detection Kit; Amersham Life Sciences, Buckinghamshire, UK), and bands were subsequently visualised (Gel DOC-It Imaging system; Bio-Rad, Hercules, CA). Equal loading in the lanes was evaluated by stripping the blots for two hours at $37^{\circ} \mathrm{C}$ and then overnight at RT (IgG Elution Buffer; Thermo Scientific, Rockford, IL) and re-probing them with GAPDH (goat polyclonal IgG) HRP-conjugated antibody. Protein levels were analysed densitometrically by ImageJ software.

\section{MicroRNA analysis}

MicroRNA for molecular analysis was obtained from peripheral blood nuclear cells (PBNCs). Cellular RNA was isolated from $3 \times 10^{6}$ nucleated cells using the mirVana Isolation Kit with organic phenol extraction (Thermo Fisher Scientific, MA, USA) according to the manufacturer's protocol. The RNA concentration was measured using an Epoch Microplate Spectrophotometer (BioTek, VT, USA), and $100 \mathrm{ng}$ was used for first-strand cDNA synthesis. For cDNA synthesis, $4 \mu \mathrm{L}$ of each sample was used. First-strand cDNA synthesis was performed in all samples using a qScript microRNA cDNA Synthesis Kit (Quantabio, MA, USA). qPCR for the assessment of miRNA expression was performed with a Bio-Rad CFX96 RealTime Detection System (Bio-Rad, CA, USA). The reaction solution consisted of $1 \mu \mathrm{L}$ of cDNA sample, iQ SYBR Green Supermix (BioRad, CA, USA), Universal Primer provided in qScript microRNA Synthesis Kit, and a forward primer specific to miRNA analysis. Quantification of the target microRNA expression value was expressed as $2^{\mathrm{Ct}}$. To find the best reference gene, the NormFinder algorithm was used; miRNA-93 was set as a reference miRNA.

\section{Statistical analysis}

The arithmetic means and S.D.s were calculated on an IBM computer using Statistica version 5.0 software (Chicago, IL, USA). Data are given mostly as the mean \pm S.D., and were analysed using the Mann-Whitney U test. Statistical significance was defined as $\mathrm{p}<0.05$.

\section{Results}

In total, 66 subjects were evaluated. The Cushing's syndrome (CS) and healthy control groups were matched for age and gender as well as well-known cardiovascular risk factors, including hypertension, history of ischaemic heart disease, myocardial infarct, cerebral stroke, peripheral artery disease, and aortic aneurysm. There were no significant differences in clinical characteristics between the study groups except for the existence of hypercortisolism in the former group.

\section{Endothelial dysfunction marker analysis}

Hypercortisolaemia is associated with increased risk of endothelial dysfunction. Endothelin-1 is a powerful vasoconstrictor peptide of small and large vessels, which also acts as a modulator of vasomotor tone. It was reported that in most CVD the circulating levels of ET-1 were increased, and ET-1 at elevated pathological concentrations acts also as proinflammatory and promotes cell proliferation, and thus attributes to the development of atherosclerosis and hypertension. Many cardiovascular complications are associated with endothelial dysfunction, which is particularly defined as dysregulation of the vascular function associated with an imbalance in the close interdependence of $\mathrm{NO}$ and ET-1 [24]. Due to difficulties in NO measurement, the analysis of ET-1 is presumably a good marker of vascular endothelial dysfunction. Thus, taking into consideration its biological and pathophysiological significance, we first analysed the expression profile of ET-1 using anti-ET-1 antibody in plasma of peripheral 

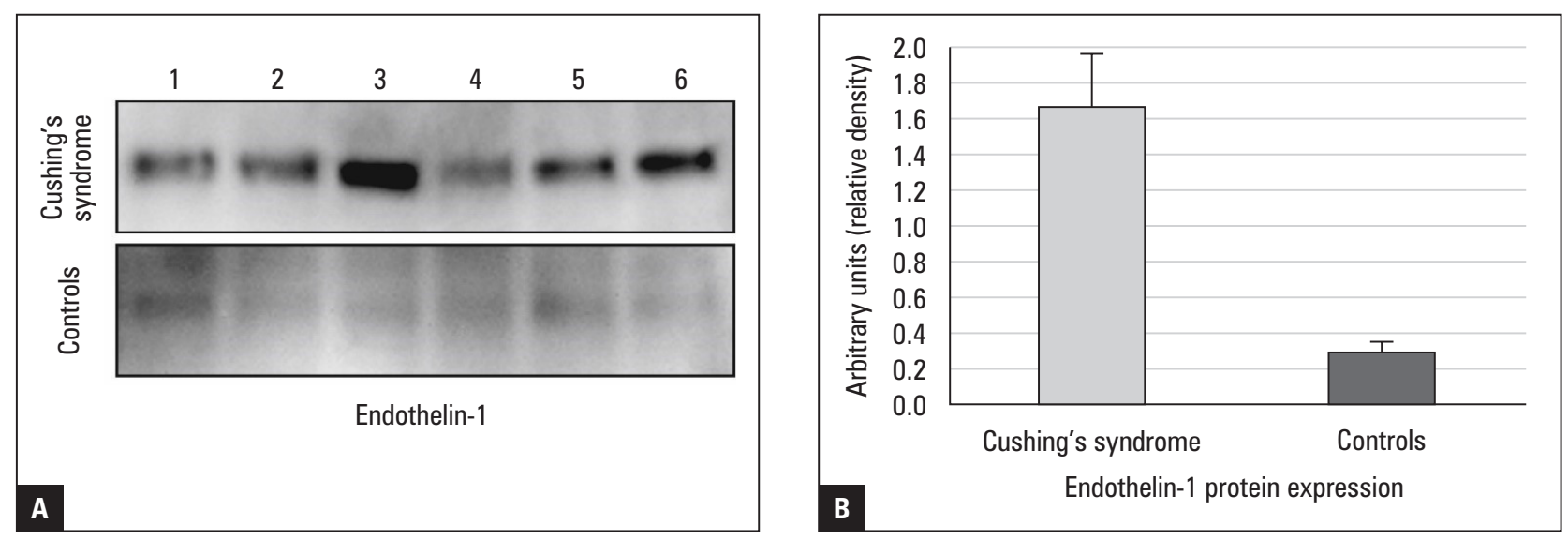

Figure 1. The expression profile of endothelium dysfunction-related molecule, ET-1, in peripheral blood from patients with Cushing's syndrome and healthy controls. The protein expression of ET-1 was determined by Western blot (A). Protein bands obtained were analysed by densitometry $(\boldsymbol{B})$. Bars represent mean \pm standard deviation $(S D)$ of selected protein to GAPDH ratios calculated in both examined groups. Representative images of the performed analyses are shown

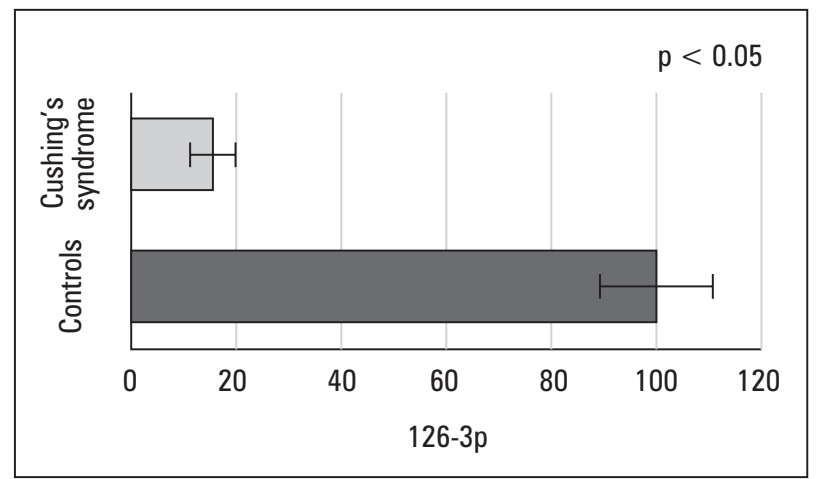

Figure 2. Cellular miRNA-126-3p profile in Cushing's syndrome (CS) patients and controls. MiRNA-126-3p was significantly down-regulated in peripheral blood nuclear cells (PBNCS) collected from patients with CS compared to healthy volunteers. The results are expressed as the percentage of the control value taken as 100\%. ${ }^{*} p<0.05$ vs. control group

blood collected from all examined subjects. As shown in Figure 1, we observed significantly increased expression of ET-1 protein in CS patients compared to healthy controls. This result indicates that patients with CS recruited to this study could develop endothelial dysfunction, and thus potentially suffer from GC-induced cardiovascular complications.

\section{MicroRNA expression profiles}

Next, we performed a quantitative analysis of the expression of the selected miRNAs in peripheral blood nucleated cells (PBNCs) of Cushing's syndrome patients and controls using qRT-PCR. We chose a panel of five miRNAs (miRNA-17-5p, -126-3p, -126-5p, -150-5p, and -223-3p). Of the five analysed miRNAs, three microRNAs (miRNA-17-5p, -126-3p, and -126-5p) showed significantly lower expression, whereas two microRNAs

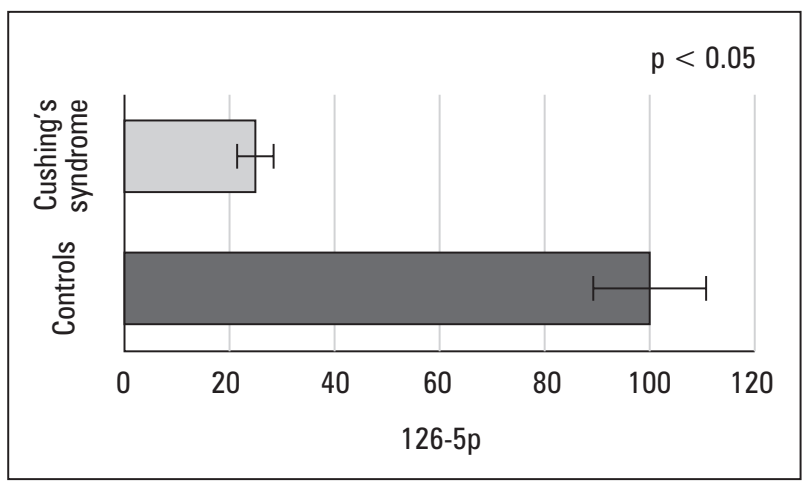

Figure 3. Cellular miRNA-126-5p profile in Cushing's syndrome (CS) patients and controls. MiRNA-126-5p was significantly down-regulated in peripheral blood nuclear cells (PBNCs) collected from patients with CS compared to healthy volunteers. The results are expressed as the percentage of the control value taken as 100\%. ${ }^{*} p<0.05$ vs. control group

(miRNA-150-5p and -223-3p) showed significantly higher expression in PBNCs circulating in the cardiovascular system of CS patients compared with the expression in these cells collected from control subjects (Fig. 2-6). These results indicate that selected "angioMIRs" are differently expressed in CS patients and healthy subjects, which may be related to the developed endothelial dysfunction present in the former group.

\section{Discussion}

This is the first study evaluating both plasma ET-1 expression profile and selected angiogenesis-related microRNA transcript quantification in cells circulating in PB from patients with active Cushing's syndrome.

The relationship between glucocorticoids and cardiovascular disorders has been extensively studied, 


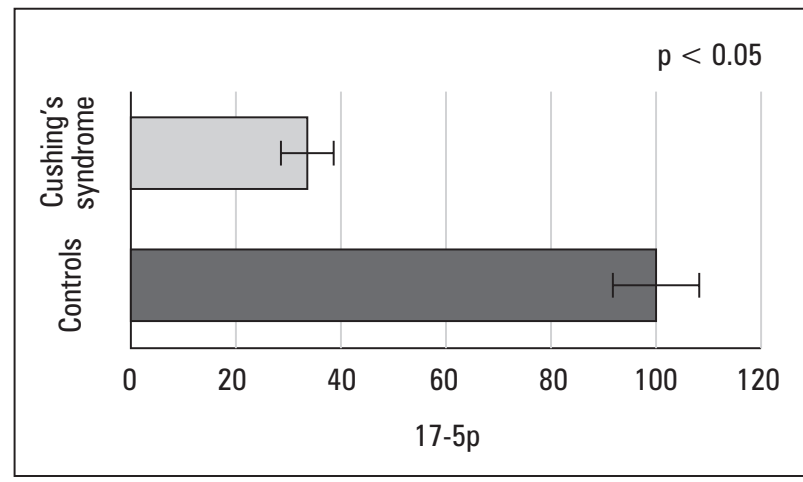

Figure 4. Cellular miRNA-17-5p profile in Cushing's syndrome (CS) patients and controls. MiRNA-17-5p was significantly downregulated in peripheral blood nuclear cells (PBNCs) collected from patients with CS compared to healthy volunteers. The results are expressed as the percentage of the control value taken as 100\%. ${ }^{*} p<0.05$ vs. control group

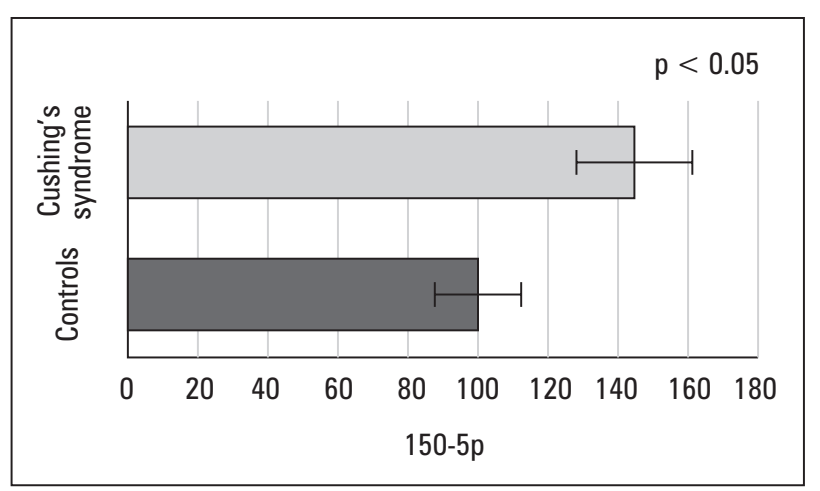

Figure 5. Cellular miRNA-150-5p profile in Cushing's syndrome (CS) patients and controls. MiRNA-150-5p was significantly upregulated in peripheral blood nuclear cells (PBNCs) collected from patients with CS compared to healthy volunteers. The results are expressed as the percentage of the control value taken as 100\%. ${ }^{*} p<0.05$ vs. control group

but with conflicting results. Some studies have shown that hypercortisolaemia is associated with increased cardiovascular morbidity and mortality, whereas other researchers cannot confirm this observation. Consistent with the previous report [8], we found significant over-expression of ET-1 activity in the PB of CS patients, which presumably indicates an overall tendency for the development of endothelial dysfunction in the cardiovascular system of subjects with CS. Patients with endogenous CS are typically exposed to high cortisol levels for longer period of time than the usual duration of laboratory experiments and interventional studies in humans, which suggests that CS could be an appropriate disease model in vivo that permits analysis of the influence of an excessive amount of GCs on the cardiovascular system and may potentially link it with GC-induced cardio-metabolic complications; thus, they

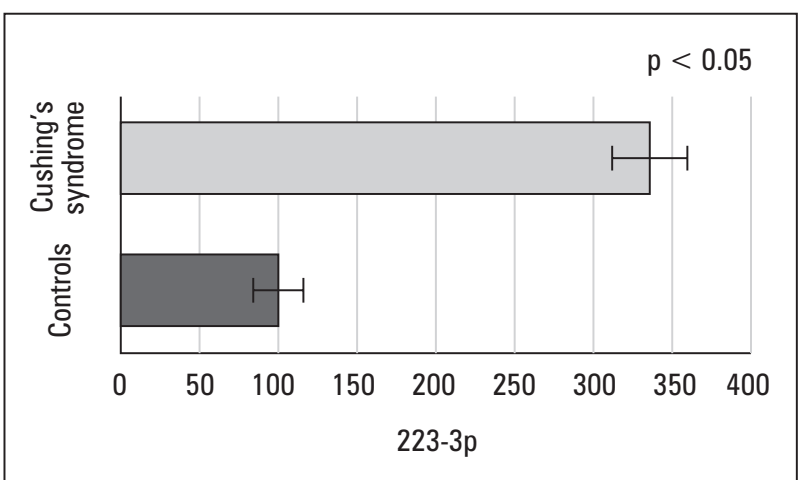

Figure 6. Cellular miRNA-223-3p profile in Cushing's syndrome (CS) patients and controls. MiRNA-223-3p was significantly upregulated in peripheral blood nuclear cells (PBNCs) collected from patients with CS compared to healthy volunteers. The results are expressed as the percentage of the control value taken as 100\%. ${ }^{*} p<0.05$ vs. control group

could be adequately investigated. Moreover, it was reported that there were no major differences between endogenous hypercortisolism in Cushing's syndrome or diet-induced cardiometabolic disorders in terms of genetic cardiometabolic risk factors and regarding the pathogenesis of their complications [25].

We found that important "angioMIRs", both miRNA-126-3p and miRNA-126-5p, were significantly downregulated in PBNC circulating in CS patients, which, according to the literature, may indicate defective angiogenesis and reduced neovascularisation as miRNA-126 stimulates angiogenesis via VEGF signalling [26]. In animal models, areas of disturbed blood flow typically present the reduced miRNA-126 expression associated with diminished endothelial cell proliferation [15]. Apart from endothelial cells, the miRNA-126 cluster plays additional roles in distinct cell types such as macrophages or endothelial progenitor cells circulating also in blood. In the latter, proangiogenic activity is associated with increased expression levels of miRNA-126, and inhibition of this expression resulted in the restriction of their proangiogenic activity [27]. Moreover, it was reported that CD34+ cells, after high glucose treatment or isolated from DM2 patients, expressed lower miRNA-126 and impaired angiogenic properties that were recovered with miRNA-126 treatment [27]. Besides, CD34+ cells from patients with cardiac insufficiency showed significantly lower expression of miRNA-126 [28]. Finally, the reduced miRNA-126 levels in monocytes from DM2 patients were associated with increased tissue factor expression and higher pro-thrombotic activity [29]. It was also found that intracellular miRNA-126 expressed in circulating platelets has also the potential to influence endothelial cells, stimulating their proliferation and inhibiting apoptosis 
via AKT signalling pathway [30]. Consequently, the intracellular downregulation of both miRNA-126-5p and miRNA-126-5p in circulating PBNCs from CS patients observed in our study are likely to contribute to the dysregulation of angiogenesis in this disease.

Consistent with previous findings in individuals with coronary artery disease and in patients with myocardial infarct [21], we determined that levels of miRNA-17 were significantly reduced and levels of miRNA-150 were upregulated in circulating PBNCs from CS patients compared to healthy subjects. The MiRNA-17 cluster controls the expression of VCAM-1, ICAM-1, and E-selectin, being involved in the coordinated manner of regulation of the inflammatory response by the complex miRNA network in endothelial cells that maintain intercellular communication with monocytic cells in the course of atherosclerosis [31]. Likewise, monocytes secrete miRNA-150, and its levels in microvesicles targeted towards endothelium increase in response to inflammatory stimuli present in atherosclerotic arteries [32]. The latter miRNA also showed the greatest discriminatory power for the diagnosis of unstable angina compared to patients with non-coronary chest pain or healthy subjects [20].

MiRNA-223 levels were also increased in circulating PBNCs from CS patients in our study. This finding is in accordance with previous studies demonstrating the significant role of intracellular miRNA-223 that can be transferred from platelets to endothelial cells. It was shown that platelet miRNA-223 has the ability to target several genes, including B1-integrin, IGF-IR, and Fbxw7, which can regulate migration and angiogenesis [30]. Furthermore, miRNA-223 was shown to have a suppressive effect on macrophage proinflammatory activation, and miRNA-223-deficient macrophages exhibited increases in M1 and decreases in M2 polarisation biomarkers [33]. Our results are in line with another study by Zampetaki et al., who showed that miRNA-223, together with miRNA-126, has a high predictive value for risk of developing acute myocardial infarct, as a result of a 10-year follow-up prospective trial [34]. Consequently, our results in GC patients could be interpreted such that the increased level of miRNA-223 might represent attempts to compensate for the above-mentioned negative effects of GC excess on endothelium-driven vascular homeostasis in a long-term exposure period in CS patients.

\section{Conclusions}

This is the first study documenting the intracellular expression of "angioMIRs" in CS patients. There were significant differences in selected miRNA expression levels in nucleated cells circulating in the peripheral blood of CS patients compared to healthy subjects. This study enriches our understanding of differential expression of miRNA related to the control of the endothelium functions in the cardiovascular system of CS patients with typical cardio-metabolic complications, and may guide towards future therapeutic interventions. Nevertheless, it requires further investigation.

\section{Acknowledgement}

This work was supported by Polish NCN grant: UMO-2011/01/B/NZ5/04224.

\section{References}

1. Barnes PJ. Glucocorticosteroids: current and future directions. Br J Pharmacol. 2011; 163(1): 29-43, doi: 10.1111/j.1476-5381.2010.01199.x, indexed in Pubmed: 21198556

2. Fardet L, Petersen I, Nazareth I. Prevalence of long-term oral glucocorticoid prescriptions in the UK over the past 20 years. Rheumatology (Oxford). 2011; 50(11): 1982-1990, doi: 10.1093/rheumatology/ker017, indexed in Pubmed: 21393338.

3. Ambroziak U, Bluszcz G, Bednarczuk T, et al. The influence of Graves orbitopathy treatment with intravenous glucocorticoids on adrenal function. Endokrynol Pol. 2017; 68(4): 430-433, doi: 10.5603/EP.a2017.0036, indexed in Pubmed: 28604944.

4. Miljic P, Miljic D, Cain JW, et al. Pathogenesis of vascular complications in Cushing's syndrome. Hormones (Athens). 2012; 11(1): 21-30, doi: 10.1007/BF03401535, indexed in Pubmed: 22450342.

5. Wierzbicka-Chmiel J, Chmiel A, Rychlik S, et al. Vascular and cardiac function in young adults with classical congenital adrenal hyperplasia. Endokrynol Pol. 2017; 68(5): 505-511, doi: 10.5603/EP.a2017.0046, indexed in Pubmed: 28879650.

6. Pivonello R, Isidori AM, De Martino MC, et al. Complications of Cushing's syndrome: state of the art. Lancet Diabetes Endocrinol 2016; 4(7): 611-629, doi: 10.1016/S2213-8587(16)00086-3, indexed in Pubmed: 27177728 .

7. Ong SLH, Whitworth JA. How do glucocorticoids cause hypertension role of nitric oxide deficiency, oxidative stress, and eicosanoids. Endocrinol Metab Clin North Am. 2011; 40(2): 393-407, ix, doi: 10.1016/j. ecl.2011.01.010, indexed in Pubmed: 21565674.

8. Kirilov G, Tomova A, Dakovska L, et al. Elevated plasma endothelin as an additional cardiovascular risk factor in patients with Cushing's syndrome. Eur J Endocrinol. 2003; 149(6): 549-553, doi: 10.1530/eje.0.1490549, indexed in Pubmed: 14640996.

9. Barbot M, Ceccato F, Scaroni C. The Pathophysiology and Treatment of Hypertension in Patients With Cushing's Syndrome. Front Endocrinol (Lausanne). 2019; 10: 321, doi: 10.3389/fendo.2019.00321, indexed in Pubmed: 31164868

10. Pereira AM, Delgado V, Romijn JA, et al. Cardiac dysfunction is reversed upon successful treatment of Cushing's syndrome. Eur J Endocrinol. 2010; 162(2): 331-340, doi: 10.1530/EJE-09-0621, indexed in Pubmed: 19933822.

11. Qin L, Zhu X, Liu X, et al. Evaluation of lipid profile and its relationship with blood pressure in patients with Cushing's disease. Endocr Connect. 2018; 7(5): 637-644, doi: 10.1530/EC-18-0010, indexed in Pubmed: 29626059.

12. Akaza I, Yoshimoto T, Tsuchiya K, et al. Endothelial dysfunction aassociated with hypercortisolism is reversible in Cushing's syndrome. Endocr J. 2010; 57(3): 245-252, doi: 10.1507/endocrj.k09e-260, indexed in Pubmed: 20023367.

13. Lamarca B. The role of immune activation in contributing to vascular dysfunction and the pathophysiology of hypertension during preeclampsia. Minerva Ginecol. 2010; 62(2): 105-120, indexed in Pubmed: 20502423.

14. Fardet L, Fève B. Systemic glucocorticoid therapy: a review of its metabolic and cardiovascular adverse events. Drugs. 2014; 74(15): 1731-1745, doi: 10.1007/s40265-014-0282-9, indexed in Pubmed: 25204470.

15. Schober A, Nazari-Jahantigh M, Wei Y, et al. MicroRNA-126-5p promotes endothelial proliferation and limits atherosclerosis by suppressing Dlk1. Nat Med. 2014; 20(4): 368-376, doi: 10.1038/nm.3487, indexed in Pubmed: 24584117.

16. Harris TA, Yamakuchi M, Ferlito M, et al. MicroRNA-126 regulates endothelial expression of vascular cell adhesion molecule 1. Proc Natl Acad Sci USA. 2008; 105(5): 1516-1521, doi: 10.1073/pnas.0707493105, indexed in Pubmed: 18227515.

17. Zampetaki A, Kiechl S, Drozdov I, et al. Plasma microRNA profiling reveals loss of endothelial miR-126 and other microRNAs in type 
2 diabetes. Circ Res. 2010; 107(6): 810-817, doi: 10.1161/CIRCRESAHA.110.226357, indexed in Pubmed: 20651284

18. Meng S, Cao JT, Zhang B, et al. Downregulation of microRNA-126 in endothelial progenitor cells from diabetes patients, impairs their functional properties, via target gene Spred-1. J Mol Cell Cardiol. 2012; 53(1): 64-72, doi: 10.1016/j.yjmcc.2012.04.003, indexed in Pubmed: 22525256

19. Wang KC, Garmire LX, Young A, et al. Role of microRNA-23b in flow-regulation of $\mathrm{Rb}$ phosphorylation and endothelial cell growth. Proc Nat Acad Sci U S A. 2010; 107(7): 3234-3239, doi: 10.1073/pnas.0914825107, indexed in Pubmed: 20133741.

20. Zeller T, Keller T, Ojeda F, et al. Assessment of microRNAs in patient with unstable angina pectoris. Eur Heart J. 2014; 35(31): 2106-2114, doi: 10.1093/eurheartj/ehu151, indexed in Pubmed: 24727883

21. Zhang R, Lan C, Pei H, et al. Expression of circulating miR-486 and miR-150 in patients with acute myocardial infarction. BMC Cardiovasc Disord. 2015; 15: 51, doi: 10.1186/s12872-015-0042-0, indexed in Pubmed: 26077801

22. Haneklaus M, Gerlic M, Kurowska-Stolarska M, et al. Cutting edge: miR-223 and EBV miR-BART15 regulate the NLRP3 inflammasome and IL-1 production. J Immunol. 2012; 189(8): 3795-3799, doi: 10.4049/jimmunol.1200312, indexed in Pubmed: 22984081.

23. Guignat L, Bertherat J. The diagnosis of Cushing's syndrome: an Endocrine Society Clinical Practice Guideline: commentary from a European perspective. Eur J Endocrinol. 2010; 163(1): 9-13, doi: 10.1530/EJE-09-0627, indexed in Pubmed: 20375177.

24. Vanhoutte PM, Shimokawa H, Feletou M, et al. Endothelial dysfunction and vascular disease. Acta Physiol (Oxf). 2009; 196(2): 193-222, doi: 10.1 111/j.1748-1716.2009.01964.x, indexed in Pubmed: 19220204.

25. Denes J, Zsippai A, Kovacs L, et al. Comparison of adipose tissue derived genes in endogenous Cushing's syndrome versus diet-induced obesity. Endokrynol Pol. 2019; 70(2): 131-134, doi: 10.5603/EP.a2018.0091, indexed in Pubmed: 30480750.

26. Nicoli S, Standley C, Walker P, et al. MicroRNA-mediated integration of haemodynamics and Vegf signalling during angiogenesis. Nature.
2010; 464(7292): 1196-1200, doi: 10.1038/nature08889, indexed in Pubmed: 20364122

27. Mocharla P, Briand S, Giannotti G, et al. AngiomiR-126 expression and secretion from circulating CD34(+) and CD14(+) PBMCs: role for proangiogenic effects and alterations in type 2 diabetics. Blood. 2013; 121(1): 226-236, doi: 10.1182/blood-2012-01-407106, indexed in Pubmed: 23144172

28. Jakob P, Doerries C, Briand S, et al. Loss of angiomiR-126 and 130a in angiogenic early outgrowth cells from patients with chronic hear failure: role for impaired in vivo neovascularization and cardiac repair capacity. Circulation. 2012; 126(25): 2962-2975, doi: 10.1161/CIRCULATIONAHA.112.093906, indexed in Pubmed: 23136161.

29. Witkowski M, Weithauser A, Tabaraie T, et al. Micro-RNA-126 Reduces the Blood Thrombogenicity in Diabetes Mellitus via Targeting of Tissue Factor. Arterioscler Thromb Vasc Biol. 2016; 36(6): 1263-1271, doi: 10.1161/ATVBAHA.115.306094, indexed in Pubmed: 27127202.

30. Elgheznawy A, Fleming I. Platelet-Enriched MicroRNAs and Cardiovascular Homeostasis. Antioxid Redox Signal. 2018; 29(9): 902-921, doi: 10.1089/ars.2017.7289, indexed in Pubmed: 28793796.

31. Zhu Ni, Zhang D, Chen S, et al. Endothelial enriched microRNAs regulate angiotensin II-induced endothelial inflammation and migration. Atherosclerosis. 2011; 215(2): 286-293, doi: 10.1016/j.atherosclerosis.2010.12.024, indexed in Pubmed: 21310411.

32. Boon RA, Vickers KC. Intercellular transport of microRNAs. Arterioscle Thromb Vasc Biol. 2013; 33(2): 186-192, doi: 10.1161/ATVBAHA.112.300139, indexed in Pubmed: 23325475.

33. Zhuang G, Meng C, Guo X, et al. A novel regulator of macrophage activation: miR-223 in obesity-associated adipose tissue inflammation. Circulation. 2012; 125(23): 2892-2903, doi: 10.1161/CIRCULATIONAHA.111.087817, indexed in Pubmed: 22580331.

34. Zampetaki A, Willeit P Tilling L et al Prospective study on circulating MicroRNAs and risk of myocardial infarction. J Am Coll Cardiol. 2012; 60(4): 290-299, doi: 10.1016/j.jacc.2012.03.056, indexed in Pubmed: 22813605 